\title{
PRIMARY LEIOMYOSARCOMA OF THE MESENTERY IN TWO SISTERS: CLINICAL AND MOLECULAR CHARACTERISTICS
}

\author{
Magdalena KoczKowska ${ }^{1 *}$, Beata S. Lipska ${ }^{1 *}$, JoAnna Grzeszewska², Janusz Limon ${ }^{1}$, \\ WOJCIECH BIERNAT ${ }^{3}$, JACEK JASSEM ${ }^{4}$ \\ *Both authors equally contributed to this work.
}

${ }^{1}$ Department of Biology and Genetics, Medical University of Gdansk, Poland

2Regional Hospital, Słupsk, Poland

${ }^{3}$ Department of Pathology, Medical University of Gdansk, Poland

${ }^{4}$ Department of Oncology and Radiotherapy, Medical University of Gdansk, Poland

\begin{abstract}
Mesenteric leiomyosarcoma (LMS) is a very rare malignancy whose familiar occurrence has not yet been reported. We present two sisters who developed intestinal LMS. Pathological analysis of the tumor samples, including evaluation of smooth muscle actin +, desmin +, Myf4-, DOG-1-, S100-, CD34- and CD117- confirmed LMS diagnosis. Molecular analysis of the lesions, both primary tumors and a liver metastasis, revealed several genomic imbalances, with recurrent chromosomal aberration: interstitial gain at chromosome 17p11.2-13.1 with the minimal overlapping region of $9.2 \mathrm{Mb}$. Our study provides further evidence for the significant role of the genes located in this region in the early stage of carcinogenesis.
\end{abstract}

Key words: mesenteric leiomyosarcoma, familial occurrence, chromosome $17 \mathrm{p}$ gain, genomic rearrangements, array-CGH.

\section{Introduction}

Leiomyosarcoma (LMS) of the mesentery is a rare malignancy and its familial occurrence has never been reported. We describe an unusual case of two sisters who developed this tumor and present a molecular analysis of both cases.

Case 1. A 62-year-old woman underwent breast-conserving therapy for T1bN0 lobular carcinoma of the left breast. Six years later at routine abdomen $\mathrm{CT}$ an irregular tumor of $54 \times 62 \times 78 \mathrm{~mm}$ adherent to the jejunum loops and multiple hypodense liver lesions up to a diameter of $26 \mathrm{~mm}$ were noted. PET/CT confirmed increased tracer uptake. The patient underwent palliative resection of the abdominal tumor and a biopsy of liver lesions. The pathologic examination revealed the tumor composed of an expansile solid portion, with infiltration of the surrounding adipose tissue (Fig. $1 \mathrm{~A}-\mathrm{C})$. The plexiform structure was composed of spindle cells with intermediate grade of atypia. The mitotic index averaged 6/10 high power fields (HPFs). Tumor cells showed expression of smooth muscle actin (SMA) and desmin, whereas Myf4, S100, CD34, DOG-1 and CD117 were not identified. Final diagnosis of LMS, grade 2 was rendered. The patient received 3 cycles of palliative chemotherapy including doxorubicin and cisplatin; however, further therapy was discontinued due to the lack of response and poor tolerance. The patient died two years later due to disease progression.

Case 2, the younger sister, at the age of 46 years, was admitted to the hospital for extirpation of the myomatous uterus. On laparotomy a large mass was revealed in the sigmoid mesentery and was removed together with the uterus. The pathologic examination showed the tumor composed of long fascicles of spindle cells with areas of necrosis. Atypia of the cells was minimal, but the mitoses were multiple. The mitotic index was 16/10 HPFs. The tumor cells were strongly SMA-positive, with scattered cells expressing desmin, while CD34, CD117, DOG-1 and Myf4 were not identified (Fig. 1D-F). The tumor was diagnosed as LMS grade 2. No malignancy was found in the uterus, 

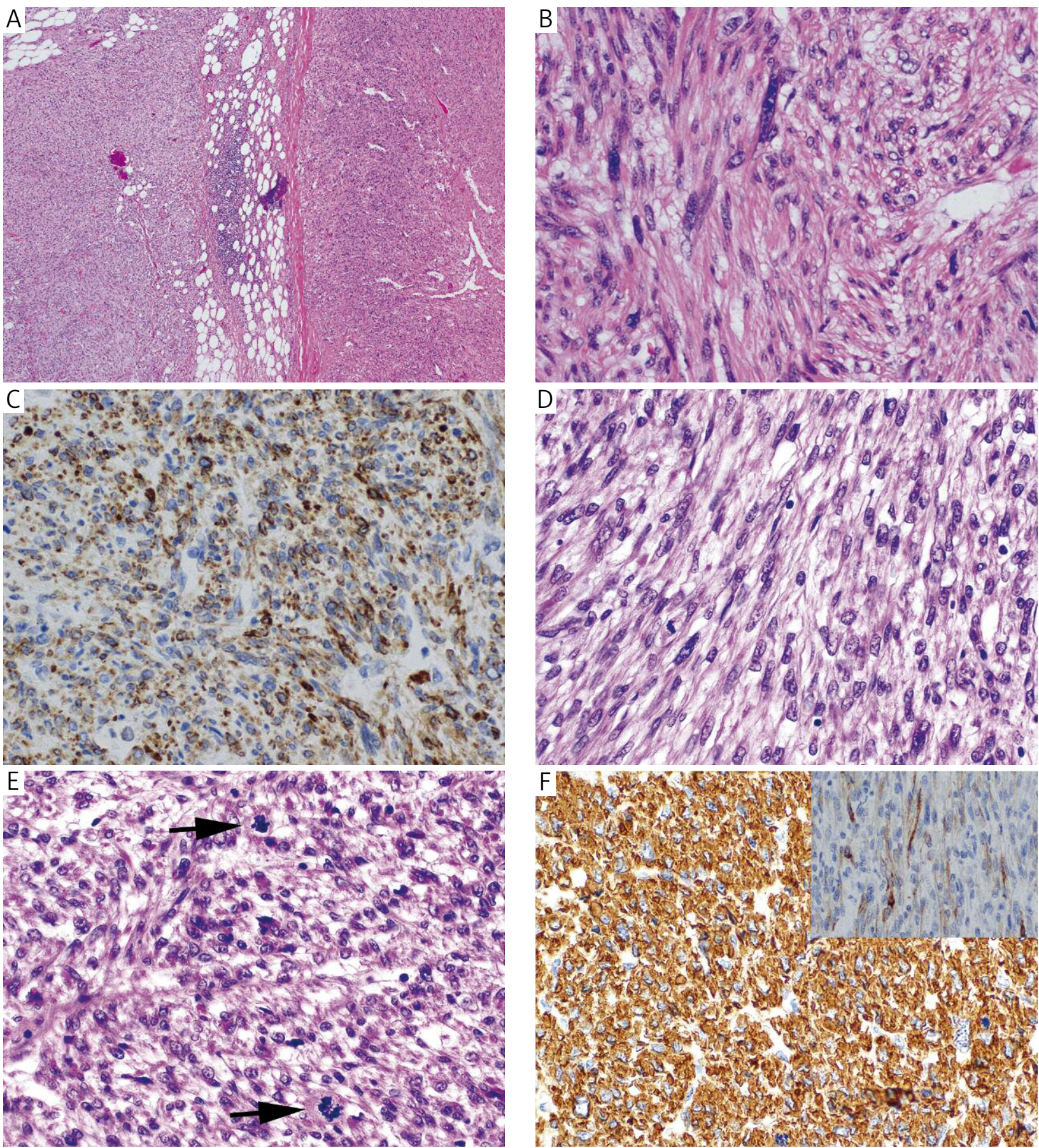

Fig. 1. A-C - patient 1. At the periphery of the main tumor bulk (A, on the right) infiltrating LMS nodules in the adipose tissue (A, on the left, magnification $20 \times$ ). The short fascicles of the elongated tumor cells contained scattered pleomorphic cells $(\mathbf{B}$, magnification $400 \times)$. Cytoplasmic expression of desmin was identified (C, magnification $400 \times)$. D-F - patient 2. The tumor was composed of long fascicles with scattered mitoses $(\mathbf{D}$, magnification $400 \times)$. Some of the mitoses were atypical (E, magnification $400 \times$, arrows). Smooth muscle actin was detected in all tumor cells (F, magnification $200 \times)$, whereas desmin was expressed by a portion of tumor cells $(\mathbf{F}$, magnification $100 \times$, inset)

apart from intramural and subserous leiomyomas. Six months later, due to extensive local recurrence, the patient underwent resection of the right colon, sigmoid, ileum and rectum. After another nine months she underwent removal of the recurrent retroperitoneal tumors. Pathologic examination in both instances sho- wed LMS. Due to further progression, the patient received two cycles of chemotherapy consisting of doxorubicin and dacarbazine, followed by one cycle of doxorubicin and cisplatin. Owing to poor tolerance and lack of response, chemotherapy was withheld. At four years from initial diagnosis she underwent chemoemboliza- 
tion of liver metastases, but eventually she died ten months later with signs of disease progression.

\section{Material and methods}

$5-\mu \mathrm{m}$ paraffin-embedded sections of primary tumors (two distinct samples from each lesion) and a liver metastasis of patient 1 were subject to molecular evaluation. DNA was extracted using a standard isolation protocol with proteinase $\mathrm{K}$ digestion, phenol-chloroform extraction and ethanol precipitation. Additionally, constitutional DNA of patient 1, as well as reference DNA for array-CGH (aCGH) and quantitative real time polymerase chain reaction (qPCR) experiments from 9 anonymous healthy female volunteers, was isolated from peripheral blood leukocytes using QIAamp DNA (Qiagen). Selected exons of KIT (exons 9, 11, 13, 17 ) and PDGFRA (exons 12, 14, 18) genes were sequenced in DNA samples extracted from all lesions. Also, TP53 mutation status was checked in the constitutional DNA sample.

Array-CGH analysis was at first performed using 3 $\times$ 720K WG-T v.3.0 Arr Del array, and later expanded using high-resolution 2.1M WG-T v2.0D Arr Del array (NimbleGen, Roche). Arrays were scanned with an MS200 Microarray Scanner and analyzed using NimbleScan and SignalMap (NimbleGen, Roche). All identified genomic imbalances were verified in the data- base of genomic variants (DGV; http://projects.tcag. ca/variation; last accessed September 2012).

\section{Results}

No germline mutation in TP53, KIT and PDGFRA genes was found. Although extraction of DNA from paraffin samples yielded genetic material of fair average quality, we were able to screen also the most common sites of somatic mutations (coverage ca. 80\%) and no mutation was detected.

Array-CGH analysis of the tumors revealed a number of aberrations, mainly terminal gains (13p, 14p) and losses (chromosomes 1p36; 4p16; 19q13), and a few interstitial deletions (3q26; 6p22, 10q22, 11q21, $\mathrm{Xq} 25$ ), with the size ranging up to $61 \mathrm{Mbp}$. Only two aberrations were present in all samples: a known $2 \mathrm{Mb}$ $\mathrm{CNV}$ at 5q13.2 (detected also in the control sample of constitutional DNA) and a duplication on the short arm of chromosome 17. The latter was found to be a recurrent somatic lesion, present in all of the analyzed neoplastic lesions, both primary and metastatic, but not in the constitutional DNA (Fig. 2). The minimal overlapping region of $9.2 \mathrm{Mb}$ covered a total of 57 genes. The TP53 gene, also located on the chromosome 17p arm, lay outside of the common region of aberration.

Even though the resolution of aCGH experiments was $5 \mathrm{kbp}$, only gross aberrations ( $>1 \mathrm{Mbp}$ ) could be

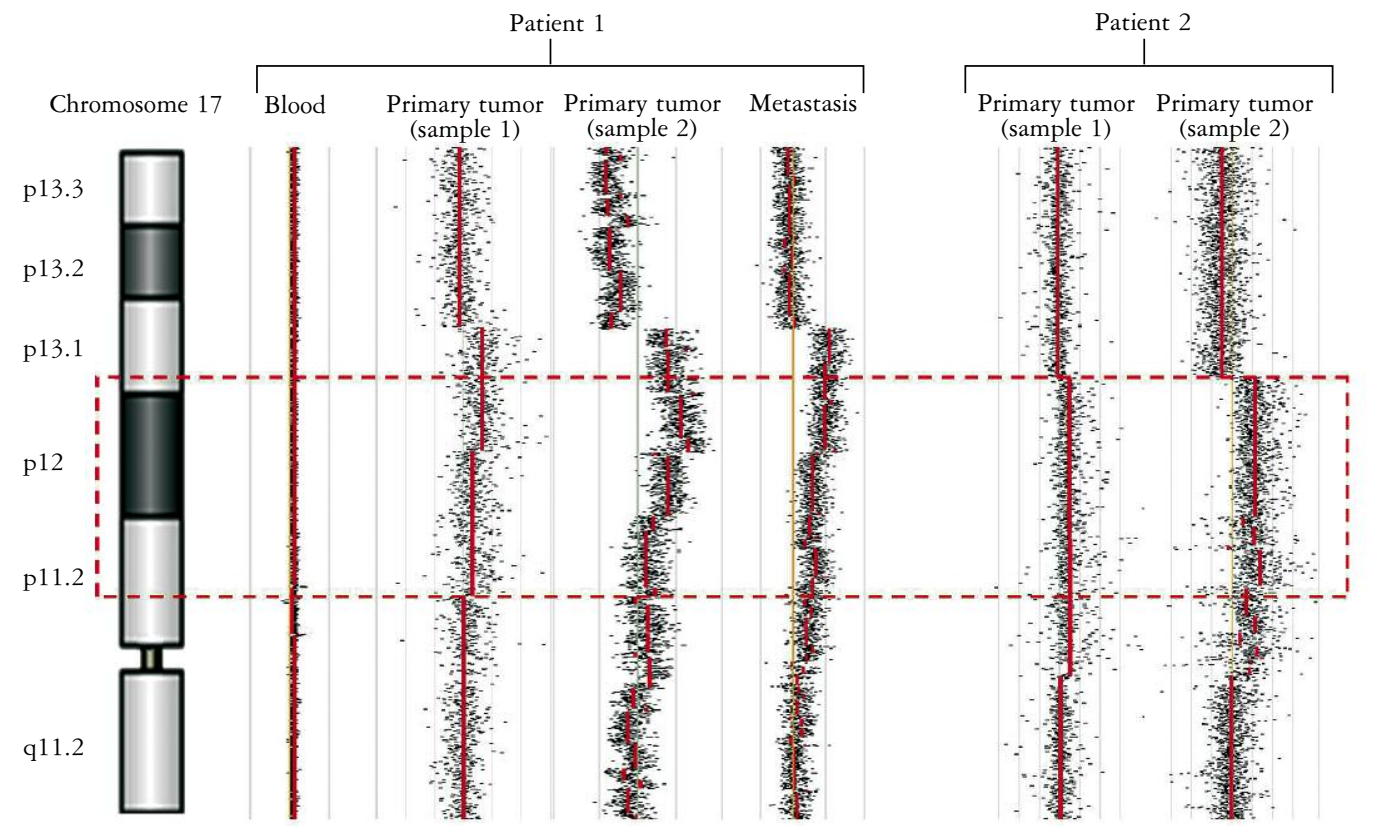

The region of gain at chromosome $17 \mathrm{p}$ detected using $720 \mathrm{~K}$ array (primary tumor sample 1 of patient 1 and primary tumor sample 1 of patient 2 ) and $2.1 \mathrm{M}$ array (the rest of the hybridizations). For patient 1, DNA extracted from neoplastic lesions (both primary and metastatic) was hybridized against constitutional DNA of the same patient. For patient 2, DNA was hybridized against a pool of control reference DNA. Log 2 ratio values for all probes are plotted as a function of their chromosomal position.

Red-dashed area: minimal overlapping region (17: 9581249-18818749 bp; GRCh36/hg 18).

Fig. 2. Array-CGH analysis showing aberration on chromosome 17 
positively detected because of the aforesaid fair average quality of DNA extracted from paraffin-embedded tissue samples. The results of microarray studies were validated by quantitative real-time PCR (Fig. 3).

\section{Discussion}

Mesenteric LMS is a rare malignancy and, since its first description in 1963 [1], only a few dozen patients have been reported [2, 3]. LMS requires differentiation from gastrointestinal stromal tumor (GIST), the most common mesenchymal malignancy of the GI tract. The older reports on mesenteric smooth muscle tumors cannot be translated into the current classification, especially those that were not analyzed regarding CD117 expression typical for GIST. Most of the cases previously diagnosed as leiomyomas, LMS and leiomyoblastomas would now be reclassified into the realm of GIST. Hence, true malignant smooth muscle tumors are currently very uncommon.

The only effective therapy for mesenteric LMS is surgery, as these tumors are notoriously resistant to radiotherapy and chemotherapy. Of the two cases reported here, one was diagnosed at a locally advanced stage and the other at dissemination; therefore neither could be effectively managed with surgery. The tumors did not show an immunophenotypic profile typical for GIST (lack of CD34, DOG1 and CD117 and expression of desmin); nor did they carry mutations in the KIT and PDGFRA genes. Thus, in neither case was imatinib therapy attempted.

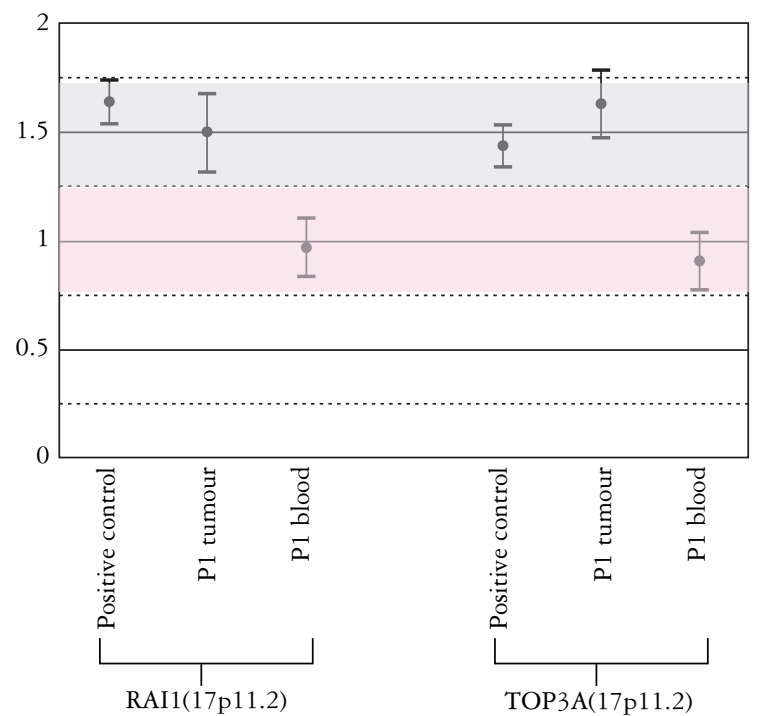

Dosage of the two genes localized in minimal overlapping region $17 \mathrm{p} 11.2: R A I 1$ and TOP3A relative to two reference genes: GPR15 (3q11.2) and ERMP1 (9p24.1) and normalized to reference DNA. Patient's constitutional DNA and tumor DNA were analyzed against a positive control DNA sample extracted from a case with constitutional duplication at $17 \mathrm{p} 11.2$ (Potocki-Lupski syndrome). All samples were run in triplicate.

The diploid (two copies of a gene) samples have the normalized ratio $=1$ (rosecolored band), whereas for triploid the normalized ratio $=1.5$ (grey-colored band).

Fig. 3. Normalized gene copy number ratios measured by qPCR
The uniqueness of our case was the occurrence of apparently identical malignancy of the small intestine mesentery in two sisters. This prompted us to perform molecular analysis of both tumors and constitutional DNA analysis. To start with, we excluded Li-Fraumeni syndrome as no TP53 germline mutation was detected. Subsequently, a search for a common genomic aberration was undertaken. Even though genomic imbalances are common in LMS, with the estimated occurrence exceeding $90 \%$, no consistent, recurrent aberrations have yet been observed at the chromosomal level [3-6]. The majority of tumors have complex karyotypes, presenting with numerous gains and losses, including an amplification at $17 \mathrm{p}$ [3-6]. Usually, numerous chromosomal aberrations are detected for a single tumor. In line with the foregoing, in the presented cases, a number of gains and losses were detected (mean 9 aberrations per sample). Nevertheless, partial $17 \mathrm{p}$ gain was the only gross somatic chromosomal anomaly detected in all lesions and, what is more, it shared $\sim 80 \%$ similarity (Fig. 2).

Chromosome $17 \mathrm{p}$ gains are presumed to be relatively common in LMS, with the estimated frequency of 33$55 \%$ [4]. Proximal 17p gains frequently occur also in other sarcomas, including osteosarcoma, chondrosarcoma and malignant fibrous histiocytoma $[3,5]$. Besides, constitutional aberrations in the $17 \mathrm{p} 11.2$ region cause four well-known constitutional genomic syndromes: Potocki-Lupski, Smith-Magenis, CharcotMarie-Tooth type 1 and hereditary neuropathy with liability to pressure palsies (HNPP). The region is one of the genomic "recombination hotspots", as it is particularly prone to rearrangements due to distinct redundancy of large, complex and highly identical lowcopy repeats, which are prone to mediate nonallelic homologous recombination.

The fact that $17 \mathrm{p}$ aberration was present in all lesions, both primary and metastatic, provides further evidence for the significant role of the genes located in this region in the early stage of carcinogenesis. Accordingly, we have attempted to further define the minimal overlapping region at $17 \mathrm{p}$ through comparison with previously reported genomic imbalances. Disappointingly, most of the published studies were performed with very low resolution, usually by means of classical karyotyping or comparative genomic hybridization; hence only large aberrations were described, making precise genotype-phenotype correlation studies almost impossible.

In the identified minimal overlapping region a total of 57 genes are located. Of these, a few have already been proposed as candidate genes in the pathogenesis of sarcoma. Two large expression profiling studies performed on LMS series identified SREBF1 [6] and $M Y O C D$ [7] genes to be highly overexpressed. SREBF1 codes for a transcription factor that regulates fatty acid and cholesterol synthesis, inhibition of which sensitizes 
resistant cells to death apoptosis [8]. MYOCD encodes transcriptional serum response factor essential for cardiac and smooth muscle development and differentiation, and its expression is typical for well-differentiated LMS [7, 9].

The development of LMS in both sisters is suggestive of an autosomal recessive trait. However - in line with Knudson's “two-hit” hypothesis - an autosomal dominant trait due to gonadal mosaicism cannot be excluded. Notwithstanding the above, further studies are needed to definitively identify the yet unknown gene responsible for cancer development.

The study was partially financed by the Polish Ministry of Science and Higher Education (grant no. N N402 2092 35).

\section{References}

1. Yannopoulos K, Stout AP. Primary solid tumors of the mesentery. Cancer 1963; 16: 914-927

2. Lazaridis Ch, Papaziogas B, Pavlidis T, et al. Leiomyosarcomas of the mesentery. Annals Gastroenterol 2001; 14: 128-131

3. Modern soft tissue pathology. Tumors and non-neoplastic conditions. Miettinen M (ed.). Cambridge University Press, Cambridge 2010.

4. Yang J, Du X, Chen K et al. Genetic aberrations in soft tissue leiomyosarcoma. Cancer Lett 2009; 275: 1-8

5. Mitelman F, Johansson B, Mertens F (eds.) Mitelman Database of chromosome aberrations and gene fusions in cancer. http://cgap.nci.nih.gov/Chromosomes/Mitelman. Accessed 29 September 2012

6. Meza-Zepeda LA, Kresse SH, Barragan-Polania AH, et al. Array comparative genomic hybridization reveals distinct DNA copy number differences between gastrointestinal stromal tumors and leiomyosarcomas. Cancer Res 2006; 66: 8984-8993.

7. Pérot G, Derré J, Coindre JM, Tirode F, et al. Strong smooth muscle differentiation is dependent on myocardin gene amplification in most human retroperitoneal leiomyosarcomas. Cancer Res 2009; 69: 2269-2278.

8. Eberhard Y, Gronda M, Hurren R, et al. Inhibition of SREBP1 sensitizes cells to death ligands. Oncotarget 2011; 2: 186-196.

9. Gibault L, Pérot G, Chibon F, et al. New insights in sarcoma oncogenesis: a comprehensive analysis of a large series of 160 soft tissue sarcomas with complex genomics. J Pathol 2011; 223: 64-71.

\section{Address for correspondence}

Jacek Jassem MD, PhD

Department of Oncology and Radiotherapy

Medical University of Gdansk

7 Dębinki St.

80-211 Gdańsk, Poland

tel. +48583492270

fax +48583492210

e-mail: jjassem@gumed.edu.pl 